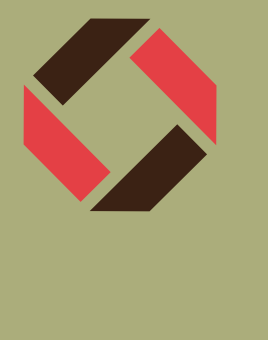

\section{Science Museum \\ in London \\ features plastics}

\section{Susan Mossman}

I n 1968 Neil Armstrong walked on the moon in a space suit made from multiple layers of polymer-based materials. This seemed the peak of modernity and was celebrated in the pop culture of the 1960s with PVC fashion wear, accessories, and furniture - evident in films such as 2001-A Space Odyssey. The Space Age and plastics seemed synonymous. We now live in an era which may see the advent of a spacecraft made with plastics and able to travel into deep space.

The importance of plastics is highlighted in the Science Museum in London by objects found in various galleries ranging from Challenge of Materials and Exploring Space to the Making of the Modern World and Health Matters. These exhibits illustrate the development of semi-synthetic plastics from the mid19th century to the fully synthetic plastics of today.

Our special exhibition Plasticity100 Years of Making Plastics (open until August 21, 2011*) tracks the importance of plastics to our lives, detailing the invention of the first entirely synthetic phenolic plastic, trade-named Bakelite, and encompassing today's plant-based plastics. Here, I tell the story of the plastics which inspired this exhibition. The exhibition itself has four sections: "The Birth of Plastics"; "Plastic Dreams," focusing on the widespread use of plastics since their introduction, and the properties and uses of key plastics including polythene, polyurethane, polyester, nylon, and acrylic; "Plastics Now," including the environmental implications of their use; and "Plastic Futures," on cutting-

* Most of the exhibits are part of the museum's permanent collection and some key items, notably the Bakelite coffin, will be redisplayed in the Challenge of Materials gallery. edge prototype products and sustainable plastics.

The foundations of the modern plastics industry lie in the mid-19th centuryan era of rich experimentation in natural and semi-synthetic materials, ranging from gutta-percha and vulcanized rubber to celluloid and milk-based casein. Lessons learned from manufacturing these materials were applied to the development of truly synthetic plastics which have existed since 1907 with the invention of Bakelite by Leo Baekeland.

The 20th century was a period of massive growth in the polymer industry. Early thermosetting polymers, the phenolics and thiourea and urea formaldehydes, were followed in the 1930s by a range of new thermoplastic plastics such as nylon, polythene, polystyrene, and polyvinyl chloride. By the 1940s, polyester appeared in both fiber and resin form. In 1946 Britain, glass-reinforced polyester, known more commonly as fiberglass, was developed and rapidly became an asset to boat, car, and furniture designers as well as inspiring new, freer forms and iconic chair shapes by key designers such as Charles Eames and Eero Saarinen.

After the Second World War, new engineered polymers were developed, providing a range of high performance materials designed for specific applications. Polyacetals and polycarbonates were developed in the 1950s, and carbon fiber-reinforced polymers in the 1960s. Plastics resin composites such as fiberglass, carbon fiber, and poly(ether ether ketone) (PEEK)-based composites defy the common understanding of polymers as low temperature materials such as these can be engineered to be used at higher and higher temperatures. This has not been without some setbacks, notably the failure of RB211 turbine fan blades made of carbon fiber.

Polymer-based composites appear in a range of applications ranging from high-tech boat hulls and Formula One racing cars to architectural moldings on historic buildings. The aerospace industry has exploited composites widelyfrom aircraft radomes and wings and

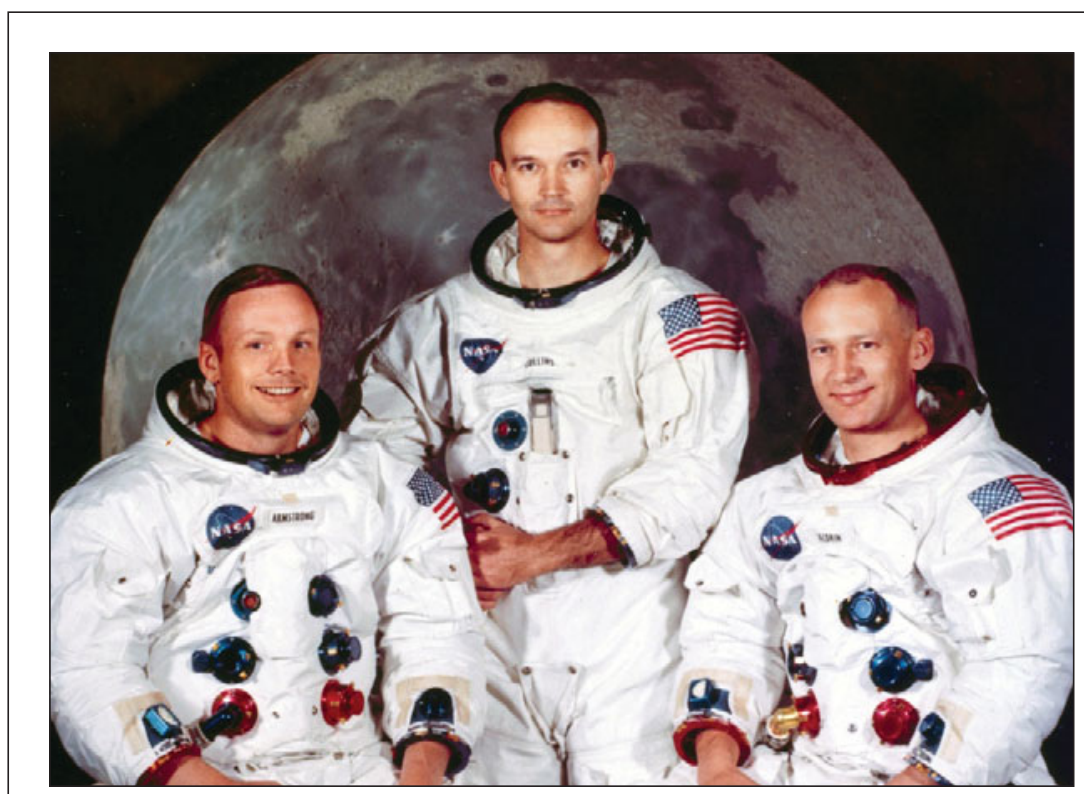

Apollo 11 Astronauts, Neil Armstrong, Michael Collins, and Edwin Aldrin, 1969. Their space suits were made from multiple layers of polymer-based materials. Source: NASA 
helicopter blades to larger scale use in modern aerospace applications. The Boeing 787 Dreamliner has a complete fuselage made of filament wound carbon fiber in an epoxy resin matrix.

Self-repairing composites are also being developed for use in aerospace applications. Defense-based organizations have worked on so-called smart systems - using composites in structures that have progressed from replicating road networks to copying nature's own blood system. This area of research known as biomimetics is becoming increasingly influential in providing new ways of thinking about materials and engineered systems.

NASA is designing a spaceship, the RXF1, made from a form of polythene, and intended for use as radiation shielding in deep space, with three times the tensile strength of aluminum, but 2.6 times lighter.

The development of copolymerscontaining mixtures of polymers such as high impact polystyrene-overcame the brittle properties of unmodified polystyrene. Block copolymers such as acrylonitrile butadiene styrene - extensively used in car bumpers - also began to play an increasing role. The block copolymer, Lycra, a polyurethane-based elastomer, became a material that has moved from fitness clothing to the fashion domain.

At the high end of sport-plasticbased materials have greatly influenced better performance whether it be in the design of carbon-fiber-based tennis racquets or Olympian athletes' clothing. The Fastskin suit worn by Olympic champion Ian Thorpe in the 2000 Sydney Olympics was made of neoprene with a surface resembling the skin of a shark designed to reduce drag in the water.

Applications in medicine range from the large scale to the nanoscale. Drug delivery systems are increasingly dependent on capsules enclosed in polymer bodies for a timed release - an example includes the Depo-Provera form of female contraceptive. Scientists at the University of Sheffield have developed polymer-based "blood" for use in emergencies, and a sample of this is displayed in the Plasticity exhibition at the Science
Museum. Polymer-based nano-machines are also being investigated using polymers which respond to their environment.

Polymers play an increasingly important role in bioengineering, whether in the form of silicone implants to replace parts lost due to disease or for cosmetic enhancement, as coatings for metals to ensure the implant is biocompatible with the body, or as polyester artificial veins and nylon sutures that replace catgut following fears of CJD. Rapidcure polymers have changed the nature of dentistry and computer-designed implants are available for those with a sufficient budget. A new area of so-called acrobatic plastics - triple-shape materials - are leading to intelligent stents-vitally important in heart and vascular surgery.

Fashion designers increasingly turn to synthetic materials and mixes for inspiration - giving the fashion wearer ever more flexibility in the form, size, and/or appearance of their clothing, with a future promising increasingly adaptive clothing. This could take the form of thermochromic clothing that lights up at different temperatures or changes color, or clothes made from shape-changing polymeric fabric that adapt to their wearers' needs or desires. Biomimetic principles have been applied to the manufacture of new materials - the earliest perhaps being Velcro - but now we have the so-called "gecko" fabrics with which the wearer can cling to sheer surfaces such as rock faces.

Automotive companies are designing concept cars that use more sustainable materials and principles - such as the 2005 i-Unit concept car by Toyota - an electric car with a leaf-shaped body made of plant-based plastics. Plant-based plastics are used for a range of applications including biodegradable packaging and mobile phone casings; however, ethical issues arise concerning the use of food crops such as corn as the feedstock for plastics, as well as issues related to genetic engineering - a sensitive issue for environmental campaigners.

Acrylics were first identified as a suitable material for corneal implants and contact lenses in the Second World War-splinters from shattered Spitfire aircraft canopies were found not to harm pilots' eyes. New porous materials for implants and contact lenses - for example, haptic lenses - are constantly being developed and refined.

In electrical applications, plastics have already played an important role as an insulating material. Although polymer-based solar cells are cheaper to make than their silicon equivalents, they are not yet as efficient so are undergoing continual improvement in performance.

Light-emitting diodes, based on organic materials, have also opened a world of increased energy-efficient and long-lived lights and ushered in a world of bendy electronic books and flexible television screens.

Work is continuing on manipulating the surfaces of polymers - whether by printing them to make them conducive 
for use in applications such as solar cells or altering the surface of polymers to make them superhydrophobic in applications such as goggles. In work inspired by beetles, superhydrophilic polymers have been developed to capture watervaluable in areas with limited water. Other polymer-based materials have inbuilt antibacterial agents - useful both in domestic contexts and in water purification applications.

In 2007 researchers at the Massachusetts Institute of Technology produced materials that repel oil (superoleophobic) and are self-cleaning without the help of soap and water. These are a form of super Teflon, as they also repel water (superhydrophobic) and are called omniphobic. This research could lead to a range of applications, including fingerprint-shedding cell-phone displays.

Packaging is a large and growing market and packaging materials can be very complex. Food or drug packing may consist of multiple layers of different polymers which results in low qual-

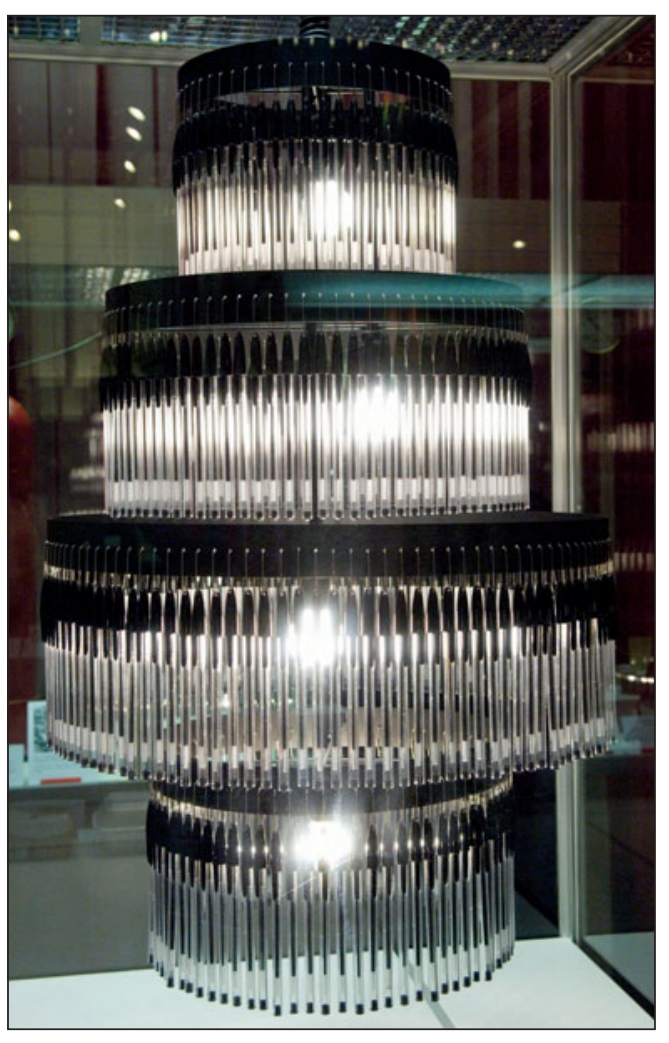

Volivik chandelier made of 347 Bic biros, made by enPieza, 2006. Source: The Science Museum ity recyclate. Packaging manufacturers are thinking increasingly about reducing and/or simplifying their products. Plastic bags that self-degrade are increasingly common and more and more customers use their own bags-often strong multiple-use woven plastic bags.

The 20th century was the era of increased consumerism - the throwaway culture that encouraged the "use once and dispose of" items as varied as toothbrushes, razors, and the bic biro (ballpoint pen)—now a design icon. In the 21 st century, we have to deal with this legacy - how are items going to be designed using plastics in a sustainable way? Designer Helen Storey took this concept to the extreme with her 2008 show displaying a range of disappearing dresses that dissolved in the raininteresting but highly impractical in the wet British climate.

Plastics are feeding the consumer market—but will this attitude have to change because of our new green credentials and emphasis on green design? Will the time for such state of the art but ultimately disposable products fade away-or will the onus be on designers to be cleverer and smarter with their design process - using the best materials for the application but ensuring good design combined with sustainability, reusability and/or repurposing?

As fuel prices increase due to scarcity or global conflictsthis will also put pressure on markets and costs of the manufacture of plastics. In 2007, 4\% of global oil production was used as feedstock for polymers and $4 \%$ for the energy to make them. Fuel shortages may well speed up imperatives for other sources of raw materials and other manufacturing methods, and potentially increase the demand for plastics for use where lighter materials are sought, for example in aerospace or automotive applications.

These pressures on developing new avenues of research are demonstrated by the new methods of recycling under development. IBM/ Stanford University teams have recently been using organic catalysts, and aiming to make endlessly recyclable plastics. Researchers at Imperial College London have managed to transform sugars found in fast growing trees and grasses into a large molecule, forming a polymer that can be used to make a compostable plastic. More recent developments include hybrid plastics incorporating algae which may be in commercial production by the end of 2010. As an alternative solution, Japanese researcher Takuzo Aida has postulated a material made of mud and glue to replace plastic.

Although plastics products have made real contributions to our quality of life in a variety of ways, mention must also be made of the obverse side. Apart from the ongoing plastics bag debate - perhaps the one most familiar to the domestic user of plastics packaging, and most frequently demonized in the press - scientists, environmental campaigners, and the press have been increasingly concerned by the issue of plastics degrading in and polluting sea water, forming so-called garbage patches. Highlighted in a recent European Commissioner's speech in April 2011, plastics form $83 \%$ of marine litter. On the positive side, steps are being made to collect and recycle these sea-going plastics waste as well as develop more suitable plastics packaging that can degrade in sea-water without deleterious effect on the environment. This is in addition to the increasingly draconian legislation covering packaging manufacturers to control such plastics waste as well as to raise public awareness of the issue. Highly political as well as emotionally charged, this is an area that cannot be ignored and which requires active and rapid solutions so that plastics can regain their good reputation - as was current in the $1920 \mathrm{~s}$ - as being a material of a thousand uses rather than a problematic material.

Materials science specialist Susan Mossman is the plastics curator at the Science Museum in London. 


\section{View, Analyze, Create in 3D*}

\section{*with the most powerful FIB and SEM}

3D NanoPrototyping

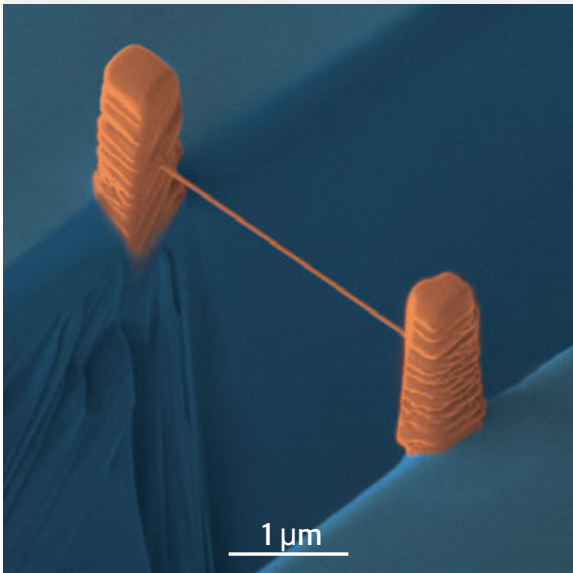

Platinum nanowire deposited and milled to about $50 \mathrm{~nm}$ diameter for use as a gas sensor

\section{Courtesy of Peter Heard,}

Bristol University, United Kingdom

Image is coloured for artistic impression
3D Analysis

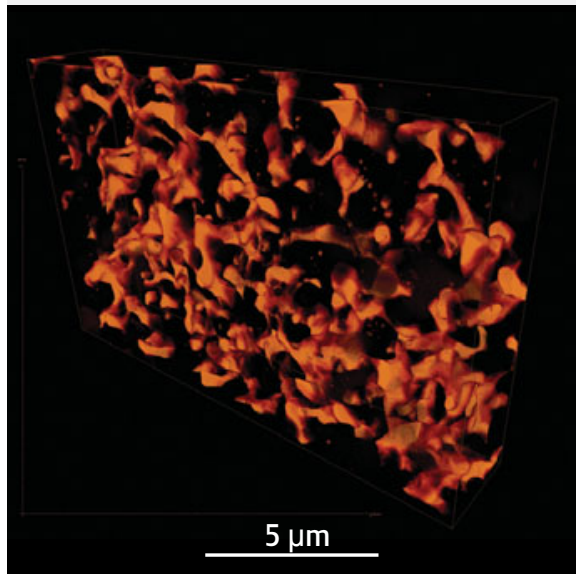

Voltex visualization of porosities in a fuel cell electrode

Courtesy of Sabanci University, Turkey
Sample preparation

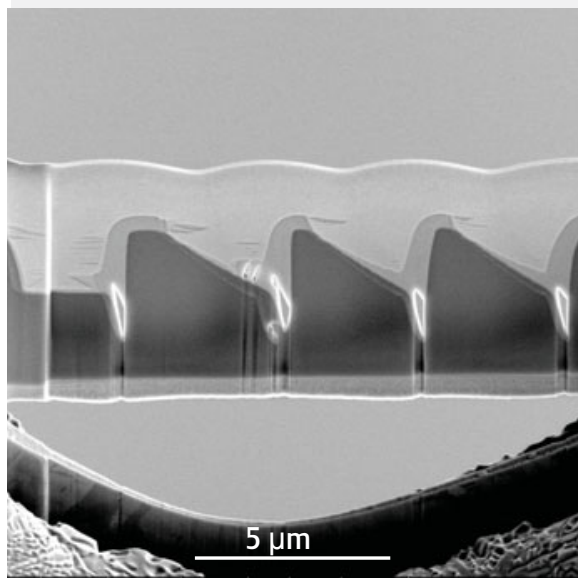

TEM lamella created to measure the amorphous damage created during FIB sample preparation

Image courtesy of D. Wall, FEI NanoPort, The Netherlands

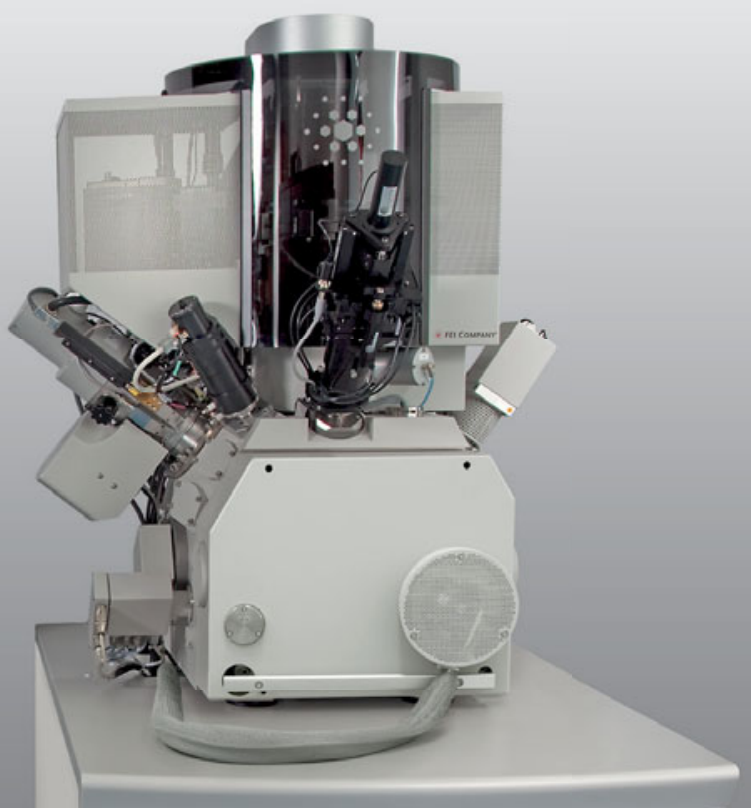

\section{Helios NanoLab ${ }^{\mathrm{TM}}$ Family}

- Best ion beam milling and unbeatable electron beam resolving power

- More contrast, even at the lowest beam currents

- Most complete, integrated suite of patterning and prototyping capabilities

- Unsurpassed thin sample preparation, 3D characterization and analysis

- Fast, easy-to-use software and automation to get the right result first time 